\section{Autoimmunity associated with Erdheim-Chester disease improves with BRAF/MEK inhibitors}

Erdheim-Chester disease (ECD) is a rare nonLangerhans cell histiocytosis characterized by the infiltration of various tissues by CD68-positive $\left(^{+}\right)$, CD1a-negative $\left(^{-}\right)$spumous histiocytes. Since the discovery that the RAS-RAF-MEK-ERK pathway is altered in almost all cases of ECD, it is now considered to be a myeloid neoplasm. Indeed, $67 \%$ of ECD patients harbor the somatic $B R A F^{\mathrm{V} 600 \mathrm{E}}$ mutation causing the constitutive activation of the BRAF-MEK pathway. ${ }^{1}$ However, the mechanisms behind the accumulation of histiocytes in ECD are not fully understood. ECD lesions typically include inflammatory cells (mainly lymphocytes) in addition to pathological histiocytes. Langerhans-cell histiocytosis (LCH) is another L-group histiocytosis, which is also considered to be a clonal disease. ${ }^{2}$ However, typical LCH lesions are inflammatory with abundant $\mathrm{CD}_{1 \mathrm{a}^{+}}$histiocytes. LCH more commonly affects children, while ECD affects adults, typically after 50 years old. ECD and LCH may be associated in so-called "mixed histiocytosis", which supports the hypothesis of a common cell of origin in these two diseases and encourages the inclusion of both of them in the L-group of the 2016 revised classification of histiocytoses.

Histiocytes, which encompass monocytes, macrophages, and dendritic cells (DC), are heterogeneous cells that play an important role in the physiology, initiation, and resolution of inflammation. Macrophages and DC are crucial for the regulation of the immune system. Autoimmunity is present in a broad spectrum of diseases in which histiocytes are pathologically altered, ranging from metabolic diseases to histiocytic disorders. Autoimmunity has also been described in myeloid neoplasms. ${ }^{4}$ In Gaucher diseases, antinuclear antibodies (ANA) are found in up to $25 \%$ of cases. ${ }^{5}$ In DestombesRosai-Dorfman disease (DRD), another rare nonLangerhans-cell histiocytosis belonging to the R-group of histiocytoses, autoimmunity has been reported in up to $20 \%$ of cases. ${ }^{6}$ There are several reports of DRD associated with systemic lupus erythematosus (SLE), ${ }^{7}$ membranous glomerulopathy, or autoimmune cytopenia. In contrast to DRD, autoimmunity has not been evaluated in L-group histiocytoses. Few cases of LCH or ECD associated with autoimmune disease (AID) have been previously reported. ${ }^{8}$ Recently, a case of polymyalgia rheumat- ica, an immune disease of unknown cause, was successfully treated with cobimetinib, an MEK inhibitor. ${ }^{9}$ BRAF and MEK inhibitors are widely used in L-group histiocytoses since they specifically target the RAS-RAF-MEKERK pathway. ${ }^{10}$ We aimed to determine the prevalence of AID and biological autoimmunity in L-group histiocytoses, and to evaluate the impact of BRAF and MEK inhibitors on the clinical and biological immune manifestations of histiocytoses.

We screened all patients with L-group histiocytoses (ECD and LCH) seen in the French National Reference Center of Histiocytoses of the Pitié-Salpêtrière Hospital, Paris. All patients had a clinical and radiological presentation compatible with L-group histiocytosis, and tissue samples were centrally reviewed for pathological confirmation. The presence of the $B R A F^{\mathrm{V} 600 \mathrm{E}}$ mutation was searched as previously described. ${ }^{11}$ The study was conducted according to the French data protection authority (CNIL) methodology reference and in accordance with the Declaration of Helsinki. Demographic, clinical, biological, imaging data, and treatments were recorded from medical charts. Patients were included if they had at least one determination of ANA [HEP-2000 slides (Immunoconcepts, Sacramento, CA, USA) with HEp$2000^{\mathrm{TM}}$ cells]. A test $>1 / 80$ was considered positive. When ANA test was positive, anti-double stranded DNA antibodies were identified with ELISA tests (ETI-dsDNA, Diasorin, Italy), and anti-extractable nuclear antibodies (ENA) were screened with ELISA (ANA screen, ORGENTEC, France), then confirmed with specific multiplex (Luminex) testing (FIDIS Connective, THERADIAG, France). Additionally, patients were all checked for the presence of other antibodies, such as anticardiolipid (ACL) antibodies, antibeta 2 glycoprotein 1 (anti $32 \mathrm{GP} 1$ ), and lupus anticoagulant (LA), in accordance with international guidelines. Antiphospholipid antibodies (APL) designed ACL antibodies, antiß2GP1 antibodies, and/or LA. For APL antibodies, two successive determinations, separated by at least 12 weeks, were required to consider the patient as "positive". We also systematically checked for the presence of AID among a list of 22 diseases (Online Supplementary Table S1). The criteria for AID were verified from medical charts and/or biological analyses, and were derived from the work of international groups. Patients were treated according to routine care. The presence of autoimmunity refers in the text to either clinical (AID) and/or biological evidence of autoimmunity. Targeted therapy, corresponding to BRAF inhibitors

Table 1. Patients of L-group histiocytosis with autoimmunity.

\begin{tabular}{lccccc} 
& $\begin{array}{c}\text { ECD } \\
(\mathbf{n = 1 6 7 )}\end{array}$ & $\begin{array}{c}\text { Mixed histiogytosis } \\
(\mathbf{n}=\mathbf{2 9})\end{array}$ & $\begin{array}{c}\text { LCH } \\
(\mathbf{n = 3 5})\end{array}$ & $\boldsymbol{p}^{* * *}$ & \\
Age at histiocytosis diagnosis, mean (SD) & $57(15)$ & $58(12)$ & $36(15)$ & $<0.0001$ & $<0.0001$ \\
Age at ANA determination, mean (SD) & $61(15)$ & $62(11)$ & $44(16)$ & $<0.0001$ & $<0.0001$ \\
\hline Sex (M/F) & $123 / 44$ & $15 / 14$ & $16 / 19$ & 0.006 & 0.001 \\
AID without ANA/APL (group 1) & $16(10 \%)$ & $0(0 \%)$ & $1(3 \%)$ & 0.091 & 0.084 \\
\hline AID with ANA/APL (group 2) & $6(4 \%)$ & $1(3 \%)$ & $0(0 \%)$ & & \\
ANA/APL/Autoantibodies without defined AID (group 3) & $47(28 \%)$ & $12(41 \%)$ & $6(17 \%)$ & & \\
Total of patients with autoimmunity & $69(41 \%)$ & $13(45 \%)$ & $7(20 \%)$ & 0.015 & 0.047 \\
\hline
\end{tabular}

AID: autoimmune disease; ANA: antinuclear antibodies; APL: antiphospholipid antibodies; APS: antiphospholipid syndrome; ECD: Erdheim Chester disease; F: female; LCH: Langerhans cell histiocytosis; n: number; M: male;SD:Standard Deviation;SGS: Sjögren syndrome; SLE: systemic lupus erythematosus. Patients with autoimmunity were classified into three groups depending on their clinical and biological immune profile: Group 1: presence of SLE, SGS, APS, or other AID without ANA and/or persistent APL; Group 2: presence of AID (except SLE, SGS and APS) with ANA and/or persistent APL; Group 3: presence of ANA, persistent APL, or other autoantibodies, without criteria for AID. *Comparison between ECD (including mixed histiocytosis) and LCH. **Comparison between three groups: AID, ANA, and APL. 
(vemurafenib and dabrafenib) and MEK inhibitors (cobimetinib and trametinib) were prescribed according to physicians' decisions, usually when the severity of the disease required. For patients treated with BRAF or MEK inhibitors, we determined the outcomes of AID and biological autoimmune parameters and noted the results of successive determinations of antiphospholipid titers. Follow up ended in August 2018. Comparisons were performed with Student $t$-test, ANOVA, the Kruskal-Wallis test, Wilcoxon matched pairs signed-rank test, MannWhitney $U$ test, or Fisher exact test when appropriate. Survival curves were built using the Kaplan-Meier method considering the time of diagnosis of ECD to death or last follow up. All tests were two-sided; $P<0.05$ was considered statistically significant. Statistical analyses were performed using the GraphPad Prism V 6.0 (GraphPad software, La Jolla, CA, USA).

We screened 276 patients: 187 patients with ECD, 51

Table 2. Demographic, clinical, and biological characteristics of Erdheim-Chester disease patients with and without autoimmunity.

\begin{tabular}{|c|c|c|c|c|}
\hline & $\begin{array}{c}\text { All } \\
(n=196)\end{array}$ & $\begin{array}{l}\text { Autoimmunity } \\
\qquad(n=82)\end{array}$ & $\begin{array}{l}\text { No autoimmunity } \\
\qquad(n=114)\end{array}$ & $P$ \\
\hline $\operatorname{Sex}(M / F)$ & $138 / 58$ & $53 / 29$ & $85 / 29$ & 0.15 \\
\hline Age at diagnosis (mean, SD) & $57(14)$ & $58(14)$ & $57(15)$ & 0.36 \\
\hline Age at ANA dosage (mean, SD) & $61(14)$ & $62(12)$ & $60(15)$ & 0.30 \\
\hline \multicolumn{5}{|l|}{ BRAF status, n (\%) } \\
\hline $\mathrm{V} 600 \mathrm{E}$ & $\begin{array}{c}108 / 166 \\
(65 \%)\end{array}$ & $\begin{array}{l}43 / 71 \\
(61 \%)\end{array}$ & $\begin{array}{l}65 / 95 \\
(68 \%)\end{array}$ & 0.32 \\
\hline Mixed histiocytosis, n (\%) & $29(15 \%)$ & $13(16 \%)$ & $16(14 \%)$ & 0.84 \\
\hline \multicolumn{5}{|l|}{ ECD involvements, n (\%) } \\
\hline Cardiac involvement & $96(49 \%)$ & $42(51 \%)$ & $54(47 \%)$ & 0.66 \\
\hline Vascular involvement & $116(59 \%)$ & $49(60 \%)$ & $67(59 \%)$ & 1.00 \\
\hline Xanthelasma & $45(23 \%)$ & $21(26 \%)$ & $24(21 \%)$ & 0.49 \\
\hline Diabetes insipidus & $51(26 \%)$ & $22(27 \%)$ & $29(25 \%)$ & 0.87 \\
\hline CNS involvement & $69(35 \%)$ & $26(32 \%)$ & $43(38 \%)$ & 0.45 \\
\hline Retro-orbital involvement & $38(19 \%)$ & $18(22 \%)$ & $20(18 \%)$ & 0.47 \\
\hline Retroperitoneal involvement & $122(62 \%)$ & $47(57 \%)$ & $75(66 \%)$ & 0.24 \\
\hline Deaths, n (\%) & $41(21 \%)$ & $21(26 \%)$ & $20(18 \%)$ & 0.21 \\
\hline \multicolumn{5}{|l|}{ Treatments, n (\%) } \\
\hline Corticosteroids & $58(30 \%)$ & $21(26 \%)$ & $37(32 \%)$ & 0.34 \\
\hline IFN- $\alpha$ or PEG-IFN- $\alpha$ & $145(74 \%)$ & $63(77 \%)$ & $82(72 \%)$ & 0.51 \\
\hline Anakinra & $28(14 \%)$ & $9(11 \%)$ & $19(17 \%)$ & 0.31 \\
\hline Infliximab & $19(10 \%)$ & $8(10 \%)$ & $11(10 \%)$ & 1.00 \\
\hline Targeted therapy, n (\%) & $75(38 \%)$ & $32(39 \%)$ & $43(38 \%)$ & 0.88 \\
\hline Vemurafenib & $66(34 \%)$ & $28(34 \%)$ & $38(33 \%)$ & - \\
\hline Dabrafenib & $9(5 \%)$ & $4(5 \%)$ & $5(4 \%)$ & - \\
\hline Cobimetinib & $29(15 \%)$ & $11(13 \%)$ & $18(16 \%)$ & - \\
\hline Autoimmune disease, $\mathrm{n}(\%)$ & $23(12 \%)$ & $23(28 \%)$ & 0 & \\
\hline Autoimmune thyroiditis & $8(4 \%)$ & $8(10 \%)$ & 0 & \\
\hline Systemic lupus erythematosus & $3(1.5 \%)$ & $3(4 \%)$ & 0 & - \\
\hline Primary Sjogren syndrome & $3(1.5 \%)$ & $3(4 \%)$ & 0 & \\
\hline \multicolumn{5}{|l|}{ Autoimmune biology, n (\%) } \\
\hline ANA $>1 / 80$ & $50(26 \%)$ & $50(61 \%)$ & 0 & \\
\hline AntidsDNA + & $10(5 \%)$ & $10(12 \%)$ & 0 & \\
\hline AntiSS-A + & $9(5 \%)$ & $9(11 \%)$ & 0 & \\
\hline AntiSS-B + & $1(0.5 \%)$ & $1(1 \%)$ & 0 & \\
\hline APL biology* & $36(18 \%)$ & $36(44 \%)$ & 0 & \\
\hline LA & $5(3 \%)$ & $5(6 \%)$ & 0 & \\
\hline Anticardiolipid + & $31(16 \%)$ & $31(38 \%)$ & 0 & \\
\hline Anti $\beta 2 \mathrm{GP1}+$ & $7(4 \%)$ & $7(9 \%)$ & 0 & \\
\hline
\end{tabular}

M: male; F: female; IFN- $\alpha$ : interferon- $\alpha$;ANA: antinuclear antibodies; antidsDNA: antidouble stranded deoxyribonucleic acid antibodies; ECD: Erdheim-Chester disease; CNS: central nervous system; SD: Standard Deviation; APL biology: antiphospholipid biology; LA: lupus anticoagulant; ACL: anticardiolipid antibodies; anti $\beta 2$ GP1: antibeta 2 glycoprotein 1 antibodies; n: number. *Refers to LA, ACL and/or antiß2GP1 positivity. 


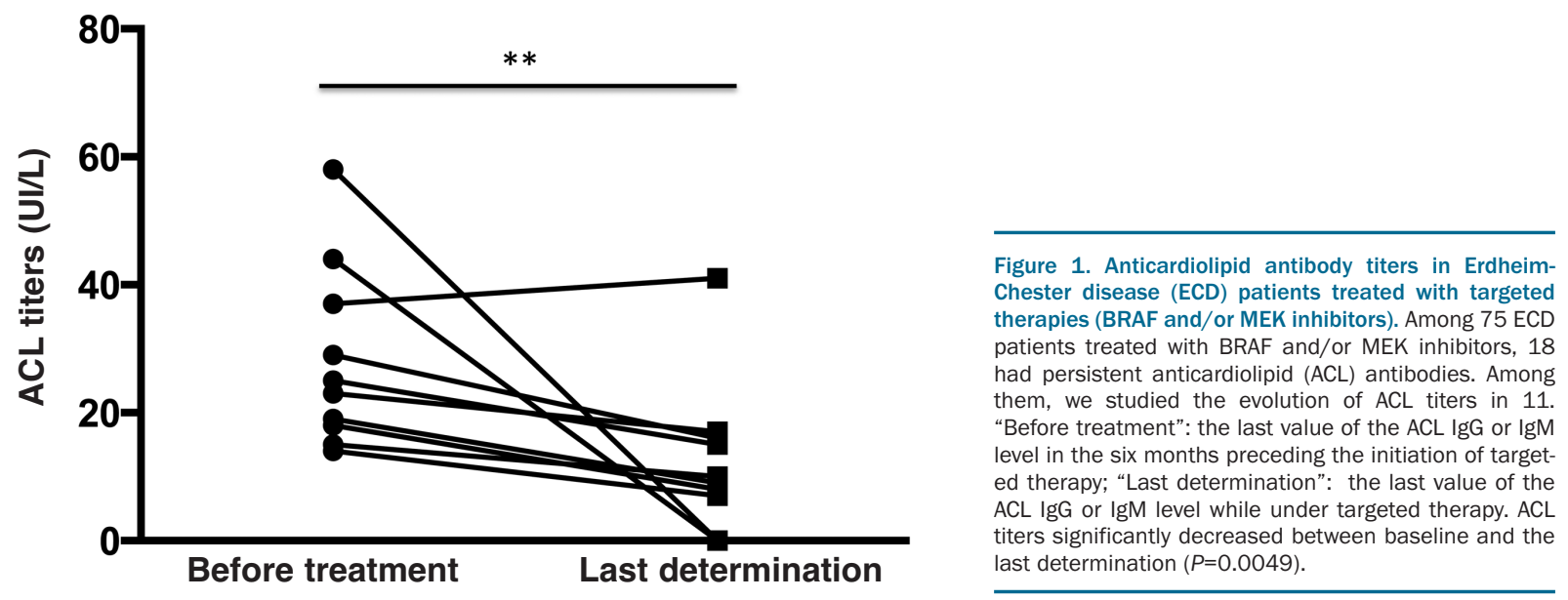

with $\mathrm{LCH}$, and 38 with mixed histiocytosis. Among them, 45 were excluded: 30 because of insufficient data in medical charts; 15 because they had no determination of autoimmune biology (Online Supplementary Figure S1). Finally, 231 patients were included in the analysis, corresponding to 167 with ECD, 35 with LCH, and 29 with mixed histiocytosis. The global prevalence of autoimmunity (biological and/or clinical) was $41 \%$ in ECD, $45 \%$ in mixed histiocytosis, and $20 \%$ in LCH (Table 1). The prevalence of autoimmunity was higher in ECD than in LCH $(42 \%$ vs. $20 \% ; P=0.015)$. The prevalence of AID was also higher in ECD than in LCH, although this difference did not reach statistical significance $(12 \%$ vs. $3 \%$; $P=0.14$ ). Among patients with ECD, there was no difference in clinical characteristics or treatments between patients with and without autoimmunity (Table 2). The $B R A F$ status was similar in patients with and without autoimmunity. There was no significant difference in survival between patients with and patients without autoimmunity. The median survival was 127 months in patients with autoimmunity (undefined in patients without autoimmunity [Hazard Ratio 1.63, (0.86-3.12); $P=0.13]$ (Online Supplementary Figure S2). Twenty-three patients out of $196(12 \%)$ had AID associated with ECD. These patients were 14 males and nine females, with a median age at ECD diagnosis of 57 years (range: 26-86 years). The AID cases included autoimmune thyroiditis $(n=8)$, primary Sjögren syndrome $(n=3)$, SLE $(n=3)$, pernicious anemia $(n=2)$, polymyalgia rheumatica $(n=2)$, APS $(n=1)$, type 1 diabetes $(n=2)$, coeliac disease $(n=1)$, autoimmune hemolytic anemia $(n=1)$, immune thrombocytopenic purpura $(n=1)$, and autoimmune alveolitis $(n=1)$. Two patients had an association of two AID: thyroiditis and pernicious anemia in one patient, coeliac disease and SLE in another.

Autoimmune disease was present before the diagnosis of ECD in 12 cases. Among them, the median time between AID and ECD diagnosis was 84 months (range: 0-336 months). The AID occurred during or after the ECD diagnosis in 11 cases. Overall, 145 patients $(74 \%)$ with ECD were treated with interferon- $\alpha$, and $19(10 \%)$ were treated with infliximab. Sixty-three patients $(43 \%)$ among those who received interferon- $\alpha$ had autoimmunity, whereas $19(37 \%)$ among those who did not receive interferon- $\alpha$ had autoimmunity $(P=0.51)$. Eight patients $(42 \%)$ had autoimmunity among those who received infliximab, and 74 patients (42\%) had autoimmunity among those who did not $(P=1.00)$. Among patients with AID and ECD treated with interferon- $\alpha$, one presented a SLE flare with multiple arthritis during the treatment, which was therefore stopped. Other patients did not experience a worsening of AID under this treatment.

Among ECD patients, 75 were treated with targeted therapy (BRAF and/or MEK inhibitor). Among patients treated with targeted therapy, six patients (8\%) had AID, $18(24 \%)$ had positive ANA, and $18(24 \%)$ had persistent antiphospholipid antibodies, including two with LA, 18 with ACL, and two with antiß2GP1 antibodies. The ACL antibody titers significantly decreased during treatment $(P=0.0049)$ (Figure 1). One patient with SLE did not have any flares during targeted therapy (17 months of follow up), whereas he experienced one flare during the six months preceding targeted therapy.

L-group histiocytoses refer to myeloproliferative neoplasms, caused by the constitutive activation of the RASRAF-MEK-ERK pathway. This leads to the qualitative and quantitative modification of DC, monocytes, and macrophages. It is possible to infer from our results that pathological histiocytes show altered functions of immune homeostasis through the modification of their phagocytosis or antigen presentation functions. Several studies demonstrated a cytokine/chemokine network in ECD lesions that may contribute to the recruitment and activation of pathological histiocytes. In ECD lesions, inflammatory cells are also present, such as eosinophil polynucleated cells and lymphocytes. Tumor necrosis factor (TNF)- $\alpha$ and TNF-receptor are increased in ECD patients compared to controls, demonstrating that TNF- $\alpha$ is an important regulator of inflammation in ECD. ${ }^{12}$ It is not clear if the modifications of these cytokines act as a cause or a consequence of the pathological changes of histiocytes. The microenvironment around pathological histiocytes, with the recruitment of immune cells, can participate in the induction of autoimmunity. An alteration of the destruction of circulating DNA by pathological histiocytes can also increase ANA occurrence. Fas ligand (FasL), an apoptosis-inducing member of the TNF cytokine family, and its receptor Fas are critical for the control of chronic immune responses and the prevention of autoimmunity. FasL (Deltam/Deltam) mice, in which T cells lack membranebound FasL, succumb to SLE-like autoimmune disease and histiocytic sarcoma. ${ }^{13}$ Hereditary Fas ligand deficits have been associated with DRD but not with L-group 
histiocytosis. However, since in our study patients were not investigated for Fas dosages, we cannot exclude the possibility that an acquired or constitutional deficit could explain the association between ECD and autoimmunity. ECD patients with severe manifestations are frequently treated with BRAF or MEK inhibitors. ${ }^{14}$ Recent evidence suggests that the MEK inhibitor could be used in autoimmune or inflammatory diseases. ${ }^{9}$ The RAS kinase has two downstream effectors: the RAF-MEK-ERK and the PI3KC-AKT-mTor pathways. ERK expression is increased in several immune diseases. ${ }^{15,16}$ In our study, we showed that ACL titers significantly decreased under BRAF or MEK inhibitors. We cannot draw any conclusions about a specific effect of the MAPK pathway inhibition, or an anti-inflammatory effect on histiocytes. Interestingly, mTOR inhibitors have been proposed for ECD treatment, and were also shown efficacious in antiphospholipid syndrome. ${ }^{17,18}$ mTOR inhibitors could probably be a good choice as a first-line treatment in patients with ECD without severe manifestation and AID when interferon- $\alpha$ cannot be used. In this study, it was more difficult to draw conclusions concerning the outcomes of AID during targeted therapy or interferon- $\alpha$ due to the heterogeneity of the diseases, the short duration of targeted treatments, and the absence of a control group. Interferon- $\alpha$ can probably be safely used in patients with thyroiditis, pernicious anemia, or type 1 diabetes, but should only be used with caution in patients with SLE, as we observed a flare in one ECD patient of the cohort under this treatment.

Interestingly, IgG4-related disease has been described in association with histiocytic disorders. ${ }^{19}$ However, we did not observe this association in our cohort of patients.

This study has several limitations. First, it is a retrospective study and we can expect that some cases of AID were not mentioned in medical charts. This bias was partially controlled because the medical charts were systematically checked for a list of AID cases. However, some type 1 diabetes cases may have been misdiagnosed as type 2 , or some patients may not have mentioned a past history of AID. Another bias may be the prescription of interferon- $\alpha$ and infliximab. However, most patients received a diagnosis of AID before the prescription of such medications, and there was no difference in the proportion of patients having received these drugs between the groups with and those without autoimmunity.

In conclusion, we report a high prevalence of autoimmunity (both AID and autoimmune biology) in ECD. ACL titers significantly decreased when patients were placed under targeted therapies. These results may help to identify new therapeutic approaches in autoimmune diseases and to better understand the mechanisms of the appearance and persistence of autoantibodies.

Anaïs Roeser, ${ }^{1}$ Fleur Cohen-Aubart, ${ }^{1}$ Paul Breillat, ${ }^{1}$ Makoto Miyara, ${ }^{2}$ Jean-François Emile, ${ }^{3}$ Frédéric Charlotte, ${ }^{4}$ Jean Donadieu, ${ }^{5}$ Zahir Amoura' and Julien Haroche ${ }^{1}$

'Sorbonne Université, Assistance Publique Hôpitaux de Paris, Hôpital de la Pitié-Salpêtrière, Service de Médecine Interne 2, Centre National de Référence Maladies Systémiques Rares et Histiocytoses, Paris-75013; ${ }^{2}$ Sorbonne Université, Assistance Publique Hôpitaux de Paris, Hôpital de la Pitié-Salpêtrière, Département d'immunochimie, Paris-75013; ${ }^{3}$ EA4340, Université Versailles-Saint Quentin, Assistance Publique Hôpitaux de Paris, Hôpital Ambroise Paré, Département de Pathologie, Boulogne-92100; ${ }^{4}$ Sorbonne Université, Assistance
Publique Hôpitaux de Paris, Hôpital de la Pitié-Salpêtrière, Service d'Anatomopathologie, Paris-75013 and 'Assistance Publique Hôpitaux de Paris, Hôpital Trousseau, Service d'Hématologie Pédiatrique, Paris-75012, France

Correspondence: FLEUR COHEN AUBART

fleur.cohen@aphp.fr

doi:10.3324/haematol.2018.214007

Information on authorship, contributions, and financial \& other disclosures was provided by the authors and is available with the online version of this article at www. haematologica.org.

\section{References}

1. Haroche J, Charlotte F, Arnaud L, et al. High prevalence of BRAF V600E mutations in Erdheim-Chester disease but not in other nonLangerhans cell histiocytoses. Blood. 2012;120(13):2700-2703.

2. Haroche J, Cohen-Aubart F, Rollins BJ, et al. Histiocytoses: emerging neoplasia behind inflammation. Lancet Oncol. 2017;18(2):e113-e125.

3. Emile JF, Abla O, Fraitag S, et al. Revised classification of histiocytoses and neoplasms of the macrophage-dendritic cell lineages. Blood. 2016;127(22):2672-2681.

4. Wang JC, Sindhu H, Chen C, et al. Immune derangements in patients with myelofibrosis: the role of Treg, Th17, and sIL2Ralpha. PLoS One. 2015;10(3):e0116723.

5. Serratrice C, Bensalah N, Penaranda G, et al. Prevalence of autoantibodies in the course of Gaucher disease type 1: A multicenter study comparing Gaucher disease patients to healthy subjects. Joint Bone Spine. 2018;85(1):71-77

6. Foucar E, Rosai J, Dorfman R. Sinus histiocytosis with massive lymphadenopathy (Rosai-Dorfman disease): review of the entity. Semin Diagn Pathol. 1990;7(1):19-73.

7. Ragotte RJ, Dhanrajani A, Pleydell-Pearce J, et al. The importance of considering monogenic causes of autoimmunity: A somatic mutation in KRAS causing pediatric Rosai-Dorfman syndrome and systemic lupus erythematosus. Clin Immunol. 2017;175:143-146.

8. Gonzalez Garcia A, Callejas Rubio JL, Rios Fernandez R, Ortego Centeno N. Pulmonary Langerhans Histiocytosis: an uncommon cause of interstitial pneumonia in a patient with Sjogren syndrome. Clin Rheumatol. 2016;35(3):825-828.

9. Chan KK, Bass AR. Checkpoint inhibitor-induced polymyalgia rheumatica controlled by cobimetinib, a MEK $1 / 2$ inhibitor. Ann Rheum Dis. 2018 May 14. [Epub ahead of print]

10. Cohen Aubart F, Emile J-F, Carrat F, et al. Targeted therapies in 54 patients with Erdheim-Chester disease, including follow-up after interruption (the LOVE study). Blood. 2017;130(11):1377-1380.

11. Emile JF, Diamond EL, Helias-Rodzewicz Z, et al. Recurrent RAS and PIK3CA mutations in Erdheim-Chester disease. Blood. 2014;124(19):3016-3019.

12. Dagna L, Corti A, Langheim S, et al. Tumor necrosis factor alpha as a master regulator of inflammation in Erdheim-Chester disease: rationale for the treatment of patients with infliximab. J Clin Oncol. 2012;30(28):e286-290.

13. O'Reilly LA, Tai L, Lee L, et al. Membrane-bound Fas ligand only is essential for Fas-induced apoptosis. Nature. 2009;461(7264):659-663.

14. Haroche J, Cohen-Aubart F, Emile JF, et al. Reproducible and Sustained Efficacy of Targeted Therapy With Vemurafenib in Patients With BRAFV600E-Mutated Erdheim-Chester Disease. J Clin Oncol. 2015;33(5):411-418.

15. Kong J, Li L, Lu Z, et al. MicroRNA-155 Suppresses Mesangial Cell Proliferation and TGF-beta1 Production via Inhibiting CXCR5-ERK Signaling Pathway in Lupus Nephritis. Inflammation. 2019;42(1): 255-263.

16. Wang J, Mizui M, Zeng LF, et al. Inhibition of SHP2 ameliorates the pathogenesis of systemic lupus erythematosus. J Clin Invest. 2016;126(6):2077-2092.

17. Gianfreda D, Nicastro M, Galetti M, et al. Sirolimus plus prednisone for Erdheim-Chester disease: an open-label trial. Blood. 2015;126 (10):1163-1171

18. Canaud G, Bienaime F, Tabarin F, et al. Inhibition of the mTORC pathway in the antiphospholipid syndrome. N Engl J Med. 2014;371 (4):303-312.

19. Gianfreda D, Musetti C, Nicastro M, et al. Erdheim-Chester Disease as a Mimic of IgG4-Related Disease: A Case Report and a Review of a Single-Center Cohort. Medicine. 2016;95(21):e3625. 